\title{
Parkinson's Disease: Examining the Role of the Pedunculopontine Nucleus via Animal Models
}

\author{
Bridgett Boxley \\ Department of Behavioral Brain Sciences, University of Texas at Dallas, Richardson, USA \\ Email: bxb150830@utdallas.edu
}

How to cite this paper: Boxley, B. (2017) Parkinson's Disease: Examining the Role of the Pedunculopontine Nucleus via Animal Models. Advances in Parkinson's Disease, 6, 39-44.

https://doi.org/10.4236/apd.2017.61004

Received: January 6, 2017

Accepted: February 25, 2017

Published: February 28, 2017

Copyright $\odot 2017$ by author and Scientific Research Publishing Inc. This work is licensed under the Creative Commons Attribution International License (CC BY 4.0).

http://creativecommons.org/licenses/by/4.0/

(c) (i) Open Access

\begin{abstract}
Parkinson's disease is a neurodegenerative disease that spreads rapidly through the brain, and can influence a number of vital systems. The cause of this disease appears to be brought on by the progressive inability to produce adequate dopamine in the brain. People that suffer with Parkinson's have reported REM sleep disruption at the onset of the condition. This paper reviews several animal model lesion studies related to the Pedunculopontine Nucleus, and how it plays a role in sleep regulation following a decline in dopamine production in those with parkinsonian conditions. The goal of this paper is to elucidate the functioning of the PPN and explain the nuclei's possible role in the onset and progression of parkinsonian conditions in animal models.
\end{abstract}

\section{Keywords}

Parkinson's Disease, Neurodegeneration, REM, Sleep Change, Dopamine, Substantia Nigra, Systems, PPN, PTN, Pedunculopontine

\section{Introduction}

Parkinson's disease is a neurodegenerative disease that spreads rapidly through the brain, and can influence a number of vital systems. The cause of this disease appears to be brought on by the progressive inability to produce adequate dopamine in the brain. The drop in dopamine causes a disruption in communication from the substantia nigra to the corpus striatum that results in severe impairments. People that suffer with Parkinson's may experience an inability to control fine and gross motor movement, incontinence, memory loss, REM sleep disruption, and emotional distress, just to name a few. REM sleep disorders are of particular importance for individuals facing progressive neurodegenerative disease because it can cause a more rapid decline in health. The specific cause of this sudden decline in dopamine is unknown, but it is thought to be both envi- 
ronmental, and genetic in nature. People that develop this disease have a grim prognosis, as there are very few options currently available. As with most studies, human subjects are few and far between, so we are mostly reliant on animal studies. Although animal physiology differs from humans, the Pedunculopontine functioning is observed to be consistent across species (rats, cats, humans, monkeys) [1]. Through animal studies of various types, we have begun to develop a possible working schematic of this area's properties, and how they may help find a treatment for Parkinsonian symptoms [2].

\section{Understanding Parkinsonian Symptoms and Their Neurological Basis: Basal Ganglia}

First and foremost, Parkinson's is a disease that attacks the nervous system. The area that is most involved in the degeneration is the Basal Ganglia, or more specifically, the substantia nigra. The substantia nigra is broken down into two components known as the pars compacta and the pars reticulata. Of these two partitions, the substantia compacta are considered to be the most important dopaminergic component. In the substantia nigra, the substantia compacta projects to both direct and indirect pathway neurons in the striatum. One pathway results in excitation (direct), and the other results in inhibition (indirect). The system of excitation and inhibition run on a delicate balance of positive and negative feedback that can only be maintained properly by the basal ganglia. The dopaminergic pathway, also called the nigrostriatal pathway, goes directly from the nigra compacta to the striatum via two types of dopamine receptors. The direct pathway cells depolarize the cells in anticipation of a new dopamine charge. The indirect pathway cells hyperpolarize the cell in response to the action potential. This dual pathway system results in two pathways of excitation that need to remain balanced in order to produce movement. When one pathway becomes favored over the other, compensation begins to take place. Other areas of the brain remain abnormally excited, which keeps the opposing pathway inhibited. In the case of Parkinson's disease, the indirect pathway remains hyperpolarized. The causes the thalamus to also remain inhibited which results in an inability to initiate movement.

\section{Understanding Parkinsonian Symptoms and Their Neurological Basis: PPN}

One interesting finding that could mean a better treatment for Parkinson's suffers is that of the Pedunculopontine (PPN, PPT, PPTg) area as regulatory nuclei. The specific role this area plays in the onset and progression of Parkinson's is still unclear, however, there has been much research in the area to try to understand its functioning. First, the Pedunculopontine nucleus (PPN) is an area located caudal to the substantia nigra and adjacent to the cerebellar peduncle.

In order to understand the implications of the PPN's functioning, it is important to understand its communications. The PPN receives reciprocal communications that span from the brain stem, up into the cortex. Major areas that the 
PPN communicates with are the basal ganglia, somatosensory and motor cortex, subthalamic nucleus, and the thalamus. Although communication pathways have been empirically established, it is still difficult to determine the primary communication mean of the PPN. For example, the PPN receives inhibitory afferents from the output nuclei of basal ganglia from the $\mathrm{SNr}$ and EP and the excitatory projections from the subthalamic nucleus [3]. The PPN also projects excitatory communication to the substantia pars compacta (but does not receive). The way in which the PPN communicates with the Basal Ganglia is of particular importance to this review. Unlike the substantia nigra, its neurons are primarily glutamatergic and cholinergic. We will revisit these two points later in the literature review.

\section{REM Sleep Disruption Parkinson's Disease in Humans}

There are many signs that may point to the onset of Parkinson's disease. Tremors, loss of fine motor control, loss of olfaction, changes in gait and motion, bowel disturbance, soft speaking, paralyzed facial expression, and stooping posture are all early symptoms. These symptoms, independent of each other or in any combination, can also point to a number of other physiological ailments [4]. Unfortunately, at the current state of medical diagnosis, by the time the patient is exhibiting these symptoms, they've already lost almost half of their dopaminergic pathway.

The reason for examining REM sleep disorders above all other symptoms of Parkinson's is because it is the single best predictor of neurodegenerative disease [5]. Specifically, a type of REM sleep disorder known as REM Sleep Behavior Disorder, causes the individual to not achieve atonia while in REM sleep, causing the person to act out their dreams. An example of this would be a person that frequently sleep walks. Although sleep may be initiated in the brain stem, it is possible that the PPN is the relay that initiates the various phases of that sleep cycle [6].

\section{REM Sleep Disruption Studies of Induced Parkinson's Like Symptoms in Animals}

When lesioning an area of the brain to check for system functioning, it is important to control for the type of lesioning that is taking place. Some studies lesion the PPN without accounting for the fact that there are both cholinergic and GABAergic neurons that comprise the PPN. This type of indiscriminate lesion will yield results that are not inconclusive. It is postulated that the most accurate way to produce Parkinson's in animal subjects is to inject them with a synthetic drug compound known as MPTP (methylphenyltetrahydropyradine). This drug (initially discovered by drug addicts) destroys the user's dopamine neurons, whilst sparring other pathways in the brain.

\section{Lesioning the PPN}

A particular study conducted by Petrovic, 2014 sought to use the proposed 
mouse model to investigate the PPN's role in regulating REM sleep cycles via the cholinergic pathway. In this study, the researchers used 33 Wistar rats, and performed a bilateral lesion of the PPN.

Following the lesion, an EEG was implanted to begin recording the brain activity. Following the period of recording, the animals were sacrificed and had their brains removed for staining. The recordings were passed through a Fourier transform to access for REM and NREM states. In addition to the filter, the researchers separated the REM sample into REM 1 (without atonia) or REM2 (with atonia). The data showed a significant change in the sensorimotor cortex when transitioning from wake to REM1 and wake to REM2. This increased activity suggests that the PPN lesion has decreased the necessary atonia to keep the animal from initiating movement while in REM sleep phase. The rapid cycling coupled with increased beta and theta wave amplitudes means that the individual is more likely to initiate stronger action potential to the sensorimotor and motor cortices. In a similar study, researchers interested in using stimulation to counter the "freezing" of parkinsonian patients used EEG data to access the patient's brain waves as they were trying to initiate movements. They found that when the patients began to "freeze" their Alpha waves peaked strongly. This is against what you would expect to find in an awake individual. Perhaps the overall change in dopamine causes a disruption in more than just the chemical makeup of the brain. Perhaps the lesion of the PPN results in damage to other areas? [7]. This next study reviews how the PPN related to areas along the striatal pathway and with its anatomically adjacent structures.

\section{Lesioning the Nigrostriatal Pathway}

So, as I have mentioned previously the PPN projects excitatory communication to the substantia pars compacta (but does not receive), but unlike the substantia nigra, its neurons are primarily glutamatergic and cholinergic. These projections are strong compared to other efferent to the compacta. A particular study conducted by Breit and colleagues sought to use the proposed mouse model to investigate the PPN's role in tipping the nigrostriatal pathway from balanced to imbalanced. In this study, the researchers used 22 Wistar rats, and lesioned their areas of interest balanced on the stereotaxic atlas for this breed. They began with a lesion to the substantia nigra compacta, and recorded the changes in firing rates of the PPN. After one week, the researchers performed a second lesion to the subthalamic nucleus, and again, recorded the activity of the PPN. Following a one-week period of recording, the researchers sacrificed the lab animals, removed the brain, and used a tyrosine hydroxylase stain to access the level of degeneration along the dopaminergic pathway.

\section{Results and Discussion}

Following the lesioning of the Nigrostriatal pathway and substantia compacta, PPN activity increased by 5 cells firing at 18 spikes per second. The variation here also increased, suggesting that the firing pattern became erratic in nature. 
What is interesting about this is that, when the compacta lesion occurred, the spikes became shorter and more rapid. Once the subthalamic nucleus was lesioned, the activity in the PPN dropped below the normal rate for the population in firing rate and interval spikes, but the number of cells that fired remained elevated, and the irregularity of firing remained. This artifact could be due to a neuroplasticity change that occurred as a result of the first lesion, or it could signal an error in the amount of tissue that was lesioned. If more than the direct pathway of the compacta was lesioned, lesioning the subthalamic nucleus as well would only blunt the PPN firing. I will revisit this in the limitations.

After lesioning the substantia compacta, the PPN began to fire rapidly. This lesion would mimic the imbalance created by the change from balanced pathways to primarily indirect pathway. The lesion to the subthalamic nucleus was able reverse the rapid fire condition of the PPN, to a semi normal state. This result, confirms what we knew of the substantia compacta and the subthalamic nucleus, but brings to light evidence of the idea of that the PPN being might be a regulatory area almost similar to the function of the LGN to the thalamus.

\section{Limitations}

Due to the nature of the lesions discussed in both studies, I would like to see the experiments replicated using a different method of inducing wide spread Parkinsonian symptoms. Although I understand the use of a lesion to examine the implications of selected areas, the use of MTPT would give a more thorough and real world model of what Parkinson like damage of the compacta would do the striatal pathway. It is possible that the PPN was thrown in to a compensatory loop based on another connection down the line. Given the number of pathways and areas that the PPN is communicating with, it is unreasonable to say that it is a regulator in and of itself. In the studies published by Breit and colleagues and Petrovich and colleaguesin which researchers chose to lesion using ibotenic acid or 6hydroxydopamine in a targeted area.

Pienarr and accompanying researchers conducted a similar animal model of Parkinson's Disease using rats. In their study, researchers induced lesion via unilateral substantia nigra pars compacta stereotaxic injection of the proteasome inhibitor Lactacystin. The researchers found that the degradation of mRNA levels remained unaltered, which suggests that the Lactacystin injection inhibited proteasome in the PPN. Proteasome is considered responsible for intracellular degradation of regulatory proteins. Although the researchers were investigating cholinergic degradation, the finding suggests a new, possibly more reliable, model of Parkinsonian effects on the PPN [2].

\section{Conclusion}

In this review, I have discussed the possible causes, symptoms, and neurological basis for Parkinson's disease. As previously stated, Parkinson's disease is a neurodegenerative disease that spreads rapidly through the brain, and can influence a number of vital systems. The drop in dopamine causes a disruption in com- 
munication between brain areas, resulting in a very uncomfortable death. People that suffer with Parkinson's suffer with the inability to control fine and gross motor movement both voluntary and involuntary, memory loss, emotional distress, and sleep disruption. REM sleep disorders are of particular importance for individuals facing progressive neurodegenerative disease because it can cause a more rapid decline in health. Although we have made great strides in understanding the "why" of this disease, we are far from understanding all of the symptoms, and even farther from establishing a cure for the disease in general. The medical and scientific community seems to be leaning towards stimulation as a means of slowing the progression of the patient's symptoms, but what we have demonstrated with this literature review is that the approach will likely need to have pharmacological intervention as well as brain stimulation to regulate the imbalances. I would even go so far as to say that a surgical lesion of overactive areas could be considered when finding a treatment.

\section{References}

[1] Alam, M. (2011) The Pedunculopontine Nucleus Area: Critical Evaluation of Interspecies Differences Relevant for Its Use as a Target for Deep Brain Stimulation. Brain, 134, 11-23. https://doi.org/10.1093/brain/awq322

[2] Pienaar, I.S., Harrison, I.F., Elson, J.L., Bury, A., Woll, P., Simon, A.K., et al. (2015) An Animal Model Mimicking Pedunculopontine Nucleus Cholinergic Degeneration in Parkinson's Disease. Brain Structure and Function, 1, 479-500. https://doi.org/10.1007/s00429-013-0669-5

[3] Breit, S., Bouali-Benazzouz, R., Benabid, A. and Benazzouz, A. (2001) Unilateral Lesion of the Nigrostriatal Pathway Induces an Increase of Neuronal Activity of the Pedunculopontine Nucleus, Which Is Reversed by the Lesion of the Subthalamic Nucleus in the Rat. European Journal of Neuroscience, 14, 1833-1842. https://doi.org/10.1046/j.0953-816x.2001.01800.x

[4] McDade, E.M., Boot, B.P., Christianson, T.J.H., Pankratz, V.S., Boeve, B.F., Ferman, T.J., Bieniek, K., Hollman, J.H., Roberts, R.O., Mielke, M.M., Knopman, D.S. and Petersen, R.C. (2013) Subtle Gait Changes in Patients with REM Sleep Behavior Disorder. Movement Disorders, 28, 1847-1853. https://doi.org/10.1002/mds.25653

[5] Iranzo, A., Tolosa, E., Gelpi, E., Molinuevo, J.L., Valldeoriola, F., Serradell, M., et al. (2013) Neurodegenerative Disease Status and Post-Mortem Pathology in Idiopathic Rapid-Eye-Movement Sleep Behaviour Disorder: An Observational Cohort Study. Lancet Neurology, 12, 443-453. https://doi.org/10.1016/S1474-4422(13)70056-5

[6] Petrovic, J. (2014) REM Sleep Diversity Following the Pedunculopontine Tegmental Nucleus lesion in Rat. Behavioural Brain Research, 271, 258-268.

https://doi.org/10.1016/j.bbr.2014.06.026

[7] Thevathasan, W. (2012) Alpha Oscillations in the Pedunculopontine Nucleus Correlate with Gait Performance in Parkinsonism. Brain, 135, 148-160. https://doi.org/10.1093/brain/awr315 
Submit or recommend next manuscript to SCIRP and we will provide best service for you:

Accepting pre-submission inquiries through Email, Facebook, LinkedIn, Twitter, etc. A wide selection of journals (inclusive of 9 subjects, more than 200 journals)

Providing 24-hour high-quality service

User-friendly online submission system

Fair and swift peer-review system

Efficient typesetting and proofreading procedure

Display of the result of downloads and visits, as well as the number of cited articles Maximum dissemination of your research work

Submit your manuscript at: http://papersubmission.scirp.org/

Or contact apd@scirp.org 